\title{
In Utero Detection of Retinoblastoma with Fetal Magnetic Resonance and Ultrasound: Initial Experience
}

\author{
Lisa B. Paquette, M.D. ${ }^{1}$ David Miller, M.D. ${ }^{2}$ Hollie A. Jackson, M.D. ${ }^{3}$ Thomas Lee, M.D. ${ }^{4}$ \\ Linda Randolph, M.D. ${ }^{5}$ A. Lynn Murphree, M.D. ${ }^{4}$ Ashok Panigrahy, M.D. ${ }^{6}$
}

${ }^{1}$ Division of Neonatology, Children's Hospital Los Angeles, Los Angeles, California

${ }^{2}$ Department of Obstetrics and Gynecology, University of Southern California, Los Angeles, California

${ }^{3}$ Department of Radiology, Children's Hospital Los Angeles, Los Angeles, California

${ }^{4}$ Division of Ophthalmology, Children's Hospital Los Angeles, Los Angeles, California

${ }^{5}$ Division of Medical Genetics, Children's Hospital Los Angeles, Los Angeles, California

6 Department of Radiology, Children's Hospital of Pittsburgh of UPMC, Pittsburgh, Pennsylvania

\begin{abstract}
Address for correspondence and reprint requests Ashok Panigrahy, M.D., Department of Radiology, Children's Hospital of Pittsburgh of UPMC, 45th Street and Penn Avenue, Pittsburgh, PA 15201 (e-mail: panigrahya@upmc.edu).
\end{abstract}

Am J Perinatol Rep 2012;2:55-62.

\begin{abstract}
Keywords

- retinoblastoma

- prenatal ultrasound

- fetal MRI

- extraocular abnormalities

Purpose Our aim was to evaluate and compare the ability of prenatal ultrasound (US) and fetal magnetic resonance imaging (MRI) to detect retinoblastoma lesions in utero. Methods Fetuses at risk for having bilateral retinoblastoma were enrolled in this prospective study. High-resolution US of the fetal eye was performed at 16 to 18 weeks' gestation, every 4 weeks until 32 weeks, then every 2 weeks until delivery. Fetal MRIs were performed every 8 weeks starting at 16 to 18 weeks of gestation. An exam under anesthesia (EUA) was performed postnatally, the gold standard of this study. Lesions were classified as being elevated or minimally elevated based upon their morphology. Results Of six fetuses suspected or confirmed to be at risk for developing bilateral retinoblastoma, one had tumors on her first postnatal EUA exam. A total of two minimally elevated lesions were seen by the EUA but not detected prenatally by imaging. One elevated lesion ( $2 \mathrm{~mm}$ in height) identified by postnatal EUA was initially identified by prenatal US. Fetal MRI did not detect any lesions.

Conclusion Both prenatal US and fetal MRI are limited in the detection of minimally elevated retinoblastoma lesions. Prenatal US appears to be more sensitive than fetal MRI in the detection of elevated retinoblastoma lesions.
\end{abstract}

Retinoblastoma is the most common intraocular malignancy of childhood. It occurs in 1/15,000 live births and there are 250 to 300 new cases in the United States each year. Forty percent of these cases are hereditary (individual possesses the $R b 1$ mutation), bilateral, and diagnosed by 15 months post- natally. For those children known to be at risk of inheriting the disease, postnatal screening by a pediatric ophthalmologist is ideal. Children not known to carry a predilection for the disease receive routine screening by their pediatrician. But, because the retinoblastoma tumor grows so quickly, with an received

January 17, 2012

accepted after revision

March 3, 2012

published online

July 16, 2012
Copyright $\odot 2012$ by Thieme Medical Publishers, Inc., 333 Seventh Avenue, New York, NY 10001, USA. Tel: +1(212) 584-4662.
DOI http://dx.doi.org/ 10.1055/s-0032-1316465. ISSN 2157-6998. 
accepted doubling time of 7 days, at least $35 \%$ of these tumors are advanced in their growth by term birth. Therefore, detecting these tumors prenatally may enhance prompt diagnosis and treatment, potentially avoiding enucleation and/or vision loss. Additionally, once retinoblastoma has spread outside of the globe, the prognosis is "dismal." Diagnosis and staging of retinoblastoma in the child is made by indirect ophthalmoscopy, ocular ultrasound (US), complete physical examination, blood and cerebrospinal fluid testing, and magnetic resonance imaging (MRI). ${ }^{1-4}$ Computed tomography (CT) is typically avoided for fear of ionizing radiation contributing to tumor growth. ${ }^{2,3,5,6}$ Prenatal detection of tumors may also help with prenatal family counseling and streamline coordinated postnatal oncologic management for the affected neonate.

Recent advances in imaging technology have enabled evaluation of the fetus to become more accessible and the information gained exquisitely more detailed. Specifically, fetal MRI and fetal US are being increasingly used for prenatal diagnosis. Since 1958, fetal US has become so much a part of the routine examination that 90 to $100 \%$ of pregnant women in some countries will have at least one US. ${ }^{7,8}$ In 2001, 67\% of pregnant women in the United States had an US. ${ }^{7}$ The use of fetal MRI was first reported in 1988 but fetal motion artifact limited useful information. ${ }^{9}$ In the ensuing years, faster imaging sequences like single-shot fast-spin echo have been developed that can help with reducing fetal motion artifact, one of the most common causes for poor image quality in fetal MRI.

There have been very few previously reported cases of detection of retinoblastoma in the fetus. Few studies have prospectively addressed the use of prenatal US for the detection of retinoblastoma. ${ }^{10-12}$ No prospective studies have evaluated the use of fetal MRI for the detection of retinoblastoma in the fetus. Most of our knowledge about the use of US and MRI in the evaluation of the orbit in retinoblastoma has come from postnatal experience. ${ }^{1-6}$

Our multidisciplinary team for maternal and fetal health designed a prospective study to evaluate the ability of both prenatal US and MRI to detect retinoblastoma lesions by serially scanning patients who had a fetus at risk for developing retinoblastoma. We hypothesized that the ability of fetal US and MRI to detect prenatal retinoblastoma hyperechoic lesions would depend on both size ( $>1$ to $2 \mathrm{~mm}$ in height; - Fig. 1) and morphology (minimally elevated versus elevated). We hypothesized that both imaging modalities would have difficulty with detecting smaller, flatter (minimally elevated) lesions compared with larger more elevated (nodular-like) lesions. We also hypothesized that fetal MRI would be better in detecting extraocular intracranial abnormalities. We describe our initial results from this prospective study.

\section{Methods}

This prospective study was approved by the Institutional Review Board. Subjects were referred for enrollment from ophthalmology clinic or were self-referred. The inclusion

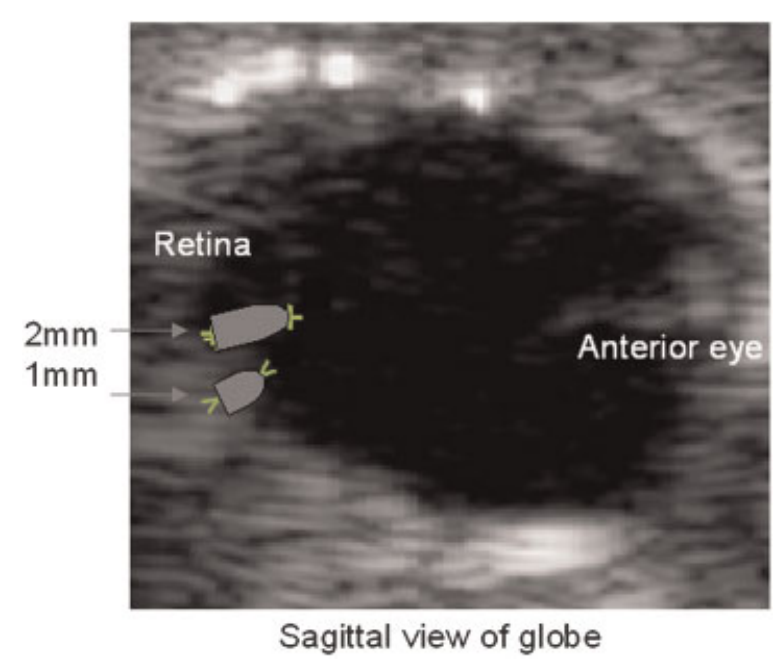

Figure 1 Hypothesized view of 1-mm (minimally elevated) and 2-mm (elevated) tumors projecting from retina as seen by fetal ultrasound.

criterion for this study was having a risk factor for retinoblastoma, which included the following: (1) the mother or father having bilateral retinoblastoma and (2) a significant family history of multiple generations of retinoblastoma. Genetic testing was offered for each parent and/or fetus. Enrollment occurred once consent was obtained. If the patient's fetus did not possess the $R b 1$ germline mutation, they were excluded from the study once the genetic testing results were available.

Fetal US was performed every 4 weeks after the initial scan (16 to 18 weeks' gestation or time of enrollment, whichever was later; see $\mathbf{- T a b l e} \mathbf{1}$ for protocol). During the last trimester, US images were obtained every 2 weeks. All US were performed by the same perinatologist on a Phillips Voluson 730 Expert. The US examination included standard evaluation of fetal anatomy, standardized view of the fetal eyes, Bmode, 3-D volume, sagittal/coronal/longitudinal 2-D measurements, lens diameter, intraocular distance, and tumor height and width (if applicable).

Fetal MRIs were performed every 8 weeks after the initial scan (same time as initial US scan). For the majority of the dataset, a 1.5-T GE magnet (General Electric Milwaukee, WI) was used with a standard torso-posteroanterior (PA) body

Table 1 Prospective Prenatal Imaging Protocol

\begin{tabular}{|l|l|l|}
\hline Gestational Age (wk) & Ultrasound & Fetal MRI \\
\hline $16-20$ & + & + \\
\hline 24 & + & \\
\hline 28 & + & + \\
\hline 32 & + & \\
\hline 34 & + & \\
\hline 36 & + & + \\
\hline 38 & + & \\
\hline 40 & + & \\
\hline
\end{tabular}

MRI, magnetic resonance imaging. 
coil, which was placed over the mother's abdomen and multiple single-shot $\mathrm{T} 2$ fast-spin echo images were obtained using 3-mm thickness (0-mm gap) in at least two orthogonal planes with a repetition time (TR)/echo time (TE) of 5 to 12/70 milliseconds, one signal acquired, a flip angle of 150 to 180 degrees, and a matrix of $128 \times 320$. The high-resolution hydrographic technique included the following parameters: $\mathrm{TE}=488$ milliseconds, $\mathrm{TR}=4000$ milliseconds, matrix $=256 \times 192$, and thickness of $3 \mathrm{~mm}$. A smaller separate subset of cases were scanned using sensitivity-encoding techniques on a 1.5-T Phillips scanner (Philips Medical Systems, Netherlands) using an eight-channel sensitivity encoding torso-PA body coil. In this group, a half-Fourier single-shot turbo spin echo sequence was performed with a TE $=120$ milliseconds, TR $=12500$ milliseconds, matrix $=232 \times 192$, and thickness of $3 \mathrm{~mm}$ (gap $=0$ ). The hydrographic sequence was performed with a $\mathrm{TE}=650$ milliseconds, $\mathrm{TR}=4000$ milliseconds, SENSE factor $=2$, matrix $=328$ to 164 , and thickness of 2 to $3 \mathrm{~mm}$ (gap $=0$ ).

Postnatal ophthalmologic exams under anesthesia (EUA) were performed by pediatric ophthalmologists. Lesions were classified as minimally elevated if they were flat or plaquelike by morphology and elevated if they were protruding from the

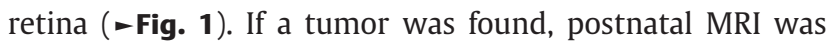
obtained.

For postnatal imaging, patients were scanned on a 1.5-T magnet and all had the same protocol for imaging the brain and orbits: axial T1 fluid-attenuated inversion recovery (TR $=2300$ milliseconds, TE $=\min , \mathrm{TI}=750$ milliseconds, field of view $($ FOV $)=22 \mathrm{~cm}$ and matrix $=256 \times 160$, NEX $=2)$; sagittal T1 FLAIR (TR $=2100$ milliseconds, $\mathrm{TE}=\mathrm{min}, \mathrm{TI}=750$ milliseconds, FOV $=22 \mathrm{~cm}$, and matrix $=256 \times 160, \mathrm{NEX}=2)$; axial $\mathrm{T} 2$ fast-spin echo $(\mathrm{TR}=6000, \mathrm{TE}=85, \mathrm{FOV}=22 \mathrm{~cm}$, matrix $=320 \times 160, \mathrm{NEX}=2)$; axial FLAIR $(\mathrm{TR}=9000, \mathrm{TE}=120$, $\mathrm{TI}=22,000$ milliseconds, $\mathrm{FOV}=22 \mathrm{~cm}$, matrix $=256 \times 160$, NEX = 1). Postcontrast axial T1 spin echo, postcontrast sagittal T1 spin echo; the following dedicated sequences for orbital imaging were performed: precontrast fat-saturated axial and coronal spin echo T1 thin section imaging of the orbits was obtained (TR $=725$ milliseconds, $\mathrm{TE}=$ min, section thickness $=3 \mathrm{~mm}$ ); postcontrast fat-saturated axial and coronal spin echo T1 thin-section images of the orbits were obtained; in addition, thin-section fat-saturated axial T2 images of the orbit were also obtained. All fetal and postnatal MRIs were read by two pediatric neuroradiologists with 7 years of experience with reading fetal MRI. Consensus was obtained for the ability to detect potential retinoblastoma lesions in the fetal MRI cases.

\section{Results}

\section{Clinical and Genetic Information of Patient Population}

There have been six patients enrolled to date. Three had questionable genetic risk (one with half-sibling affected, one with affected sibling who was later determined to have unilateral $\mathrm{Rb}$, and one whose father had unilateral $\mathrm{Rb}$ but many cousins had the disease as well). DNA testing was not performed on these three fetuses. Their prenatal and postnatal evaluations revealed no evidence of retinoblastoma lesions. Of the remaining three, one had fetal DNA demonstrating the presence of the germline mutation. The fetus identified to have the germline $R b$ mutation developed retinoblastoma tumors, which were seen on the first postnatal EUA exam and suspected on prenatal US. One of the tumors was elevated in shape $(n=1)$, and the other two were minimally elevated. Please see - Table 2 for a summary of findings.

\section{Prenatal US Findings}

Prenatal US images detected one tumor. This tumor was an elevated tumor 2 to $3 \mathrm{~mm}$ in height in the left eye at 37 weeks' gestation (-Fig. 2). At the postnatal EUA (2 months of age), the ophthalmologist described a 2-mm elevated tumor in the same eye. The minimally elevated tumors that were detected on EUA that affected the other eye were not seen on prenatal US. There were no cases in which clear images of the orbits were not obtained.

\section{Fetal MRI Findings}

Fetal MRI did not detect any minimally elevated or elevated retinoblastoma tumors, including retrospective review of the one confirmed elevated tumor that was identified by US. There were two extraocular orbital abnormalities detected. A dacryocystocele was seen in one patient at 28 and 36 weeks' gestation. Another case was found to have microphthalmia with measurements which fell in the 5th to 10th percentile. The fetal MRI demonstrated no evidence for intracranial abnormality (including no evidence of a pineal mass or of brain parenchymal congenital malformation).

\section{Postnatal MRI Findings}

Postnatal MRI was also unable to detect minimally elevated tumors. In addition, the case in which an elevated tumor was detected by prenatal US and confirmed with EUA was not detected on postnatal MRI. However, the postnatal MRI was performed 5 months after birth and after treatment. - Figure 3 is a collection of the MRIs of our affected patient.

\section{Minimally Elevated Versus Elevated Tumors}

The suspected elevated tumor on prenatal US was measured to be $\sim 2$ to $3 \mathrm{~mm}$ in height at 37 weeks' gestation (-Fig. 2). At 2 months' postnatal age, an elevated tumor was described by ophthalmologic EUA to be $\sim 2 \mathrm{~mm}$. There were also two minimally elevated tumors in the contralateral eye found at this same exam not detected by prenatal US. Please see -Fig. 4 for retinal images of this patient. = Figure $\mathbf{3}$ is a sequential series of the MRIs of the patient who had tumors at birth, including prenatal and postnatal images.

The other neonates in our study continue to remain disease-free.

\section{Discussion}

The overall goal of this article was to describe our initial experience with a prospective research protocol evaluating and comparing the use of fetal MRI and US in the prenatal diagnosis of cases at high risk for retinoblastoma. Our initial results indicated that (1) both prenatal US and MRI may be 
58 In Utero Detection of Retinoblastoma Paquette et al.

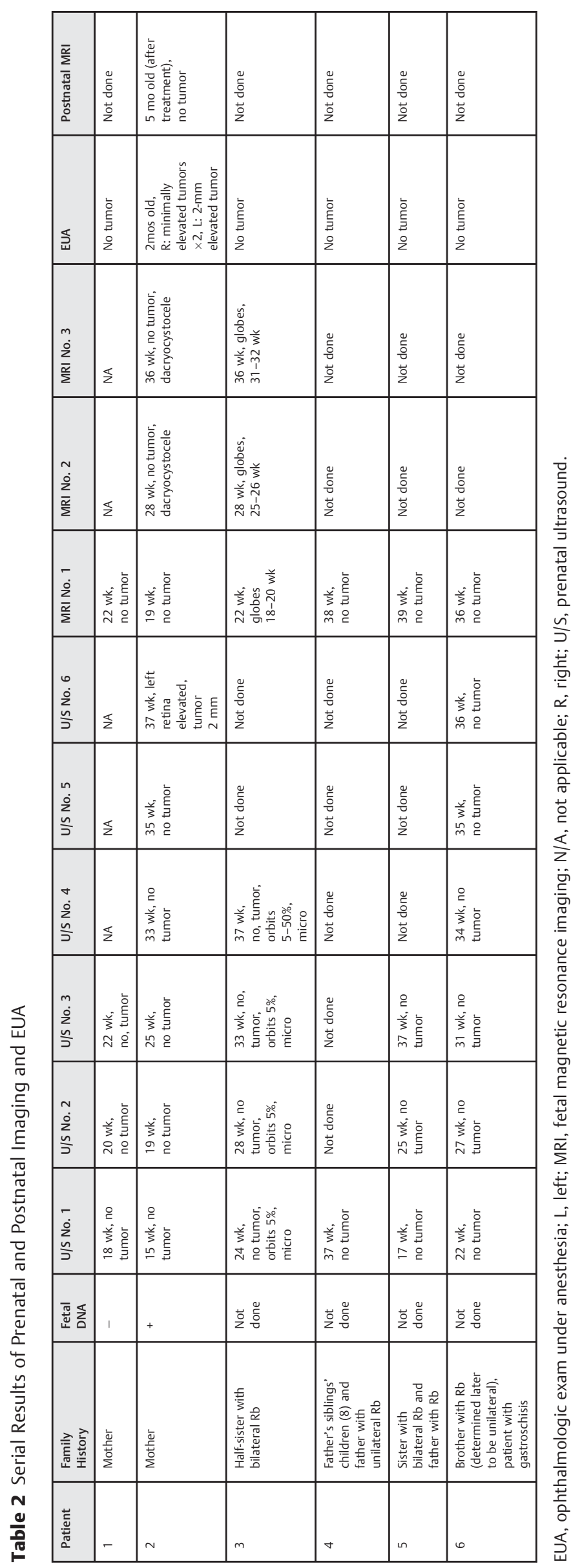

American Journal of Perinatology Reports Vol. 2 No. 1/2012 


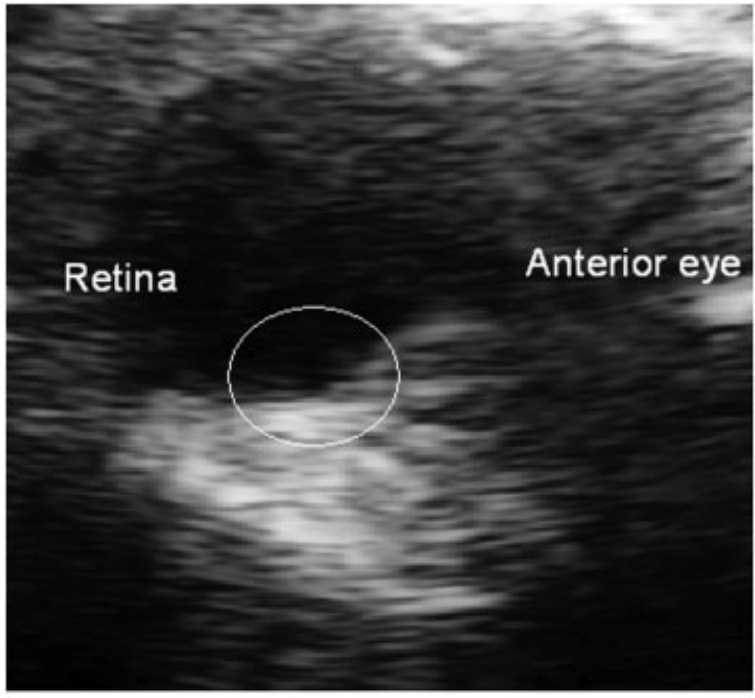

Sagittal view of globe

Figure 2 Prenatal ultrasound of patient 2. There was a suspected elevated tumor $\sim 2$ to $3 \mathrm{~mm}$ in height as seen on prenatal ultrasound at 37 weeks' gestation. insensitive to minimally elevated retinoblastoma lesions; (2) prenatal US is more sensitive to elevated lesions compared with fetal MRI; and (3) fetal MRI may be useful for diagnosis of associated extraocular findings in retinoblastoma patients. All of these finding are comparable to the more established role of postnatal US and MRI in the evaluation of retinoblastoma patients.

There have been very few previously reported cases of detection of retinoblastoma in the fetus. Two of these previously reported cases were unusually large retinoblastomas lesions. Maat-Kievit et al reported identification by US at 21 weeks of gestation of a large, face-deforming tumor. ${ }^{10}$ The tumor was described as irregularly shaped, echogenic, covered by a sonolucent area, and causing facial bone distortion. $^{10}$ Salim et al described the ultrasonographic identification of a large retinoblastoma at 38 weeks. ${ }^{11}$ This tumor was so large that it also caused deformation of the face. ${ }^{11}$ Toi et al identified a 3.7-mm lesion by US at 33 weeks of gestation, but were unable to see a $1.1-\mathrm{mm}$ or a $2-\mathrm{mm}$ retinal tumor. ${ }^{12}$ Our study did detect a 2 - to $3-\mathrm{mm}$ retinal lesion prenatally by US, which is similar to the experience of Toi et al. ${ }^{12}$ This lesion was undetectable by fetal MRI.
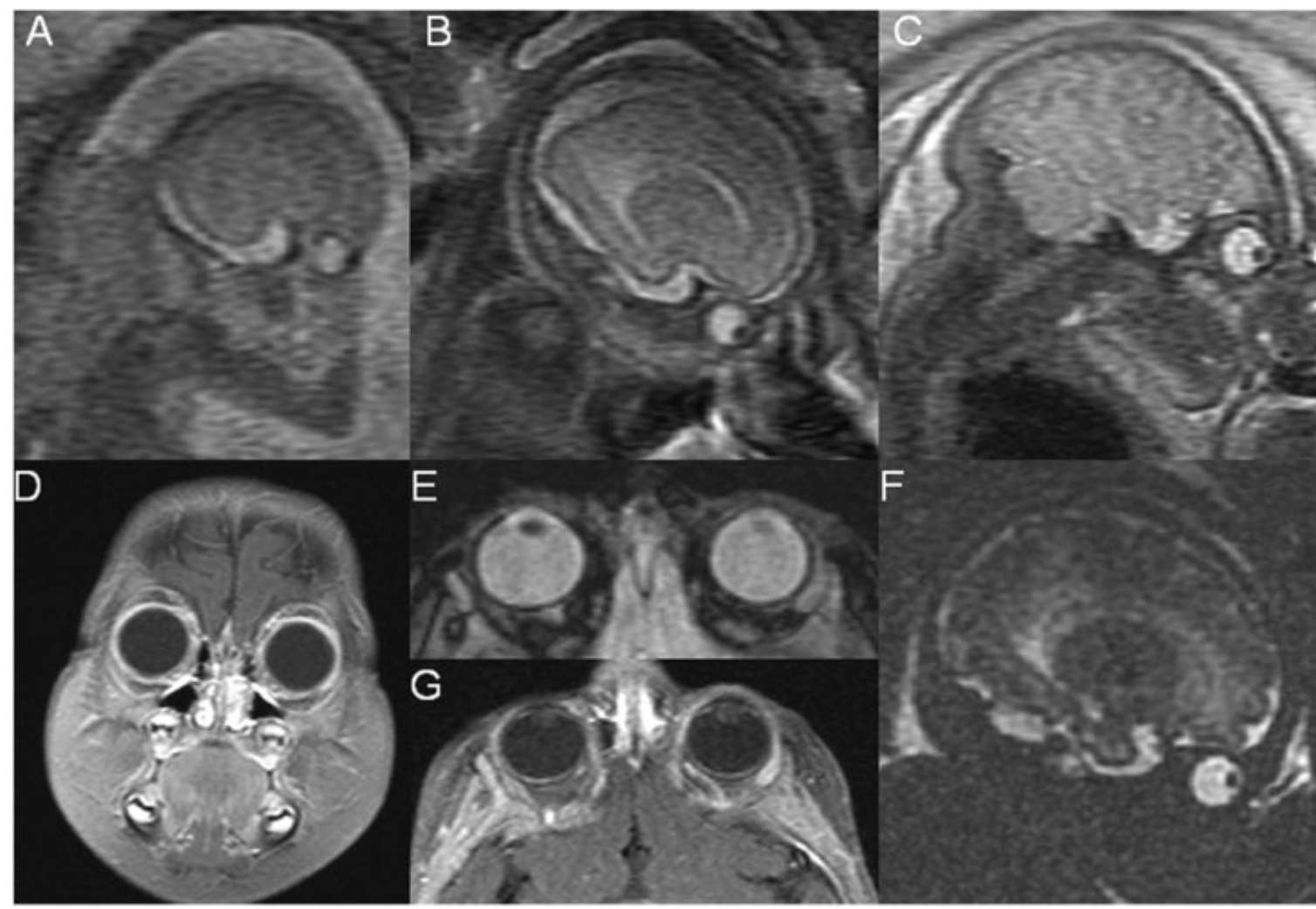

Figure 3 Fetal and postnatal magnetic resonance (MR) images of patient 2 suspected by prenatal ultrasound to have an elevated intraocular mass at 37 weeks (-Fig. 2) and confirmed on postnatal exam under anesthesia (- Fig. 4). The sequential fetal MR imaging (A-C), single-shot fast-spin echo T2 sagittal views of fetal head and orbits performed at (A) 19 weeks, (B) 28 weeks, (C) 36 weeks show no evidence of an intraocular mass. A correlating hydrographic fetal MR sequence was performed at 36 weeks $(G)$, which also confirmed no evidence of an intraocular mass. The postnatal MR imaging for this patient was performed 5 months after birth and after chemotherapy was given. No intraocular mass was detected on the postnatal MR exam (D-F), coronal fat saturated postcontrast T1 of the orbital (D), axial fat-saturated T2 imaging of the orbit (E), axial fat-saturated postcontrast $\mathrm{T} 1$ of the orbit $(\mathrm{F})$. 


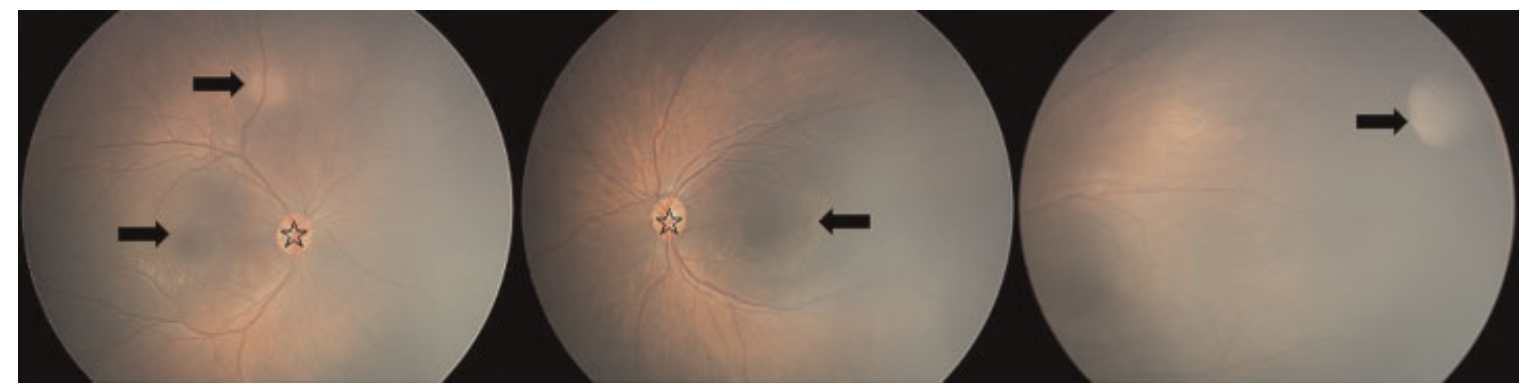

Figure 4 Postnatal exam under anesthesia of patient 2 suspected by prenatal ultrasound to have an elevated tumor. Arrow indicates tumor. Left image shows two minimally elevated (flat) tumors and middle and right images show elevated tumors. In left and middle image, optic disk designated with star.

\section{Ultrasound}

In the child, US is used for determining tumor location and extension and if calcifications are present. ${ }^{3,13,14}$ The advantages of US are that the images are dynamic and more quickly obtained than MRI or CT. ${ }^{6}$ Color Doppler imaging with US has been used to examine tumor vascularization and to differentiate tumors from hemorrhage. ${ }^{6}$

Retinoblastoma tumor growth has been documented to occur in utero ${ }^{10,11}$ and has been identified at 33 weeks of gestation in prematurely born neonates (Murphree, personal communication). However, there has only been one reported systematic attempt to identify retinoblastoma in the fetus with conventional US. ${ }^{12}$ Finger et al reported the utility of 3-D US in children to help better describe retinoblastoma tumor characteristics. ${ }^{13}$ However, no studies have reported using 3-D fetal US or fetal MRI to identify retinoblastoma. In our initial experience, fetal US, including 3-D, may be more useful in identifying elevated retinoblastoma tumors $>2 \mathrm{~mm}$ in height. This method does not detect all retinoblastoma tumors as some are minimally elevated, causing no retinal surface distortion. Based on our limited experience, lesions projecting from the retina by as little as 2 to $3 \mathrm{~mm}$ may be detected by US. The difficulty detecting smaller lesions is supported by Toi et al's experience of identifying one tumor of three out of 23 fetuses evaluated. ${ }^{12}$

\section{MRI}

In the child, MRI has been demonstrated to be useful in characterizing retinoblastomas and in differentiating the tumors from nonneoplastic lesions (Coat's disease, persistent hyperplastic primary vitreous, retrolental fibroplasia, toxocariasis, congenital hypertrophy of the retinal pigment epithelium, pigmented ocular fundus lesions as in familial adenomatous polyposis, nevus sebaceous syndrome). ${ }^{1,2,15-17}$ Specifically, heavily T2-weighted, unenhanced T1, and gadolinium-enhanced T1 images have been employed. ${ }^{1,2}$ The retinoblastoma has been found to be hypointense to vitreous on T2 and hyperintense to vitreous on $\mathrm{T} 1 .^{3}$ Tumor volumes $<0.65 \mathrm{~cm}^{3}$ have been identified postnatally. ${ }^{2}$ Additionally, endophytic growth (similar to our elevated lesions) within the retina was found to be identified by MRI accurately $68 \%$ of the time in children., ${ }^{1,2}$ It is recognized that MRI offers significant soft tissue contrast and the ability to examine a lesion in multiple planes, both of which contribute to diagnosis and treatment planning. However, the limitations with small-scale resolution and signal-to-noise ratio make describing the specific extent of tumor invasion challenging. 2,3,6

Fetal MRI has not detected any tumor, thus far. This is likely due to the inability to image items less than $3 \mathrm{~mm}$ in size. It is likely that fetal MRI may miss elevated lesions that are smaller than $3 \mathrm{~mm}$ because of the limitations with section thickness, resolution, and fetal motion: current applications using parallel imaging techniques to more quickly obtain thinner sections of the orbit may improve fetal MRI techniques. Until tissue contrasts are more easily recognized on fetal US or MRI, it is unlikely that minimally elevated retinoblastoma tumors will be identified in the fetus. Elevated tumors $>2 \mathrm{~mm}$ in height may currently be detected by prenatal US and detection by MRI may be possible in the near future with technological advances.

\section{Incidental Findings}

One of the advantages of fetal MRI is the ability to detect extraocular abnormalities that may be associated with retinoblastoma. Some of these abnormalities would include pineal lesions associated with tri-retinoblastoma. In our study, no pineal lesions were detected prenatally. Our fetal MRI scans did detect microphthalmia and a dacryocystocele. Additional possible findings that should not be confused with retinoblastoma extension would include eyelid or retinal hamartoma, hemangioma, dermoid cyst, skin tag, lid coloboma, neuromas, lenticonus, and glioma. ${ }^{18}$

\section{Management Issues}

It is likely that identifying tumors in pregnancy could lead to controversial opinions regarding management. As the doubling time of retinoblastomas is rapid, it is understandable that pursuing early delivery and oncologic treatment would be a consideration. However, there are significant risks of prematurity that must be weighed, including significant developmental delay. Delivery prior to 34 weeks would likely be unreasonable as it may preserve the globe and vision of the affected eye, but may predispose the baby to disabling global deficits. A multidisciplinary approach including ocular oncologists, perinatologists, neonatologists, and the family is essential for weighing the risks and benefits for each affected individual and their specific disease burden. 
The strength of this study was the prospective design and multidisciplinary approach, which is needed to provide correlation between prenatal US, fetal MRI, and ophthalmologic exam. Weaknesses of this study include the inability to verify tumor presence at birth as suspected by US and the small sample size. It was unfortunate that the first thorough postnatal exam of the patient in which we suspected a tumor was at 2 months postnatally. We cannot certainly discern that a tumor was present at birth or if the finding on our US was a falsepositive and the tumor grew after birth. Strengths are that this is the first systematic study to use fetal MRI to investigate the possible detection of fetal retinoblastoma. As we have a large retinoblastoma referral center, it will be possible for us to continue enrolling patients and gaining experience.

\section{Conclusion}

In conclusion, both prenatal US and fetal MRI are unable to detect fetal retinoblastoma lesions that are minimally elevated. It may be possible to detect fetal retinoblastoma tumors that are elevated and as small as 2 to $3 \mathrm{~mm}$ by US. Fetal MRI performed at 3-mm section thickness may not detect elevated lesions less than $3 \mathrm{~mm}$. Efforts to enhance diagnosis of fetal retinoblastoma may enable the initiation of treatment in a more timely fashion, which may preserve vision and/or life or prevent enucleation.

\section{Acknowledgments}

We acknowledge the assistance of Stefanie Gilinsky (genetic counselor for Retinoblastoma Center), Nancy Mansfield of the Division of Ophthalmology and Institute of Maternal and Fetal Health, Irene Klecha, Sunny Pearson, Sharon Suncin, Sheila Perez of the Institute of Maternal and Fetal Health for their assistance with recruitment and evaluation of the patients enrolled in this study. We also acknowledge Julia Castro for assistance in manuscript preparation.

\section{References}

1 Barkhof F, Smeets M, van der Valk P, et al. MR imaging in retinoblastoma. Eur Radiol 1997;7:726-731

2 de Graaf P, Barkhof F, Moll AC, et al. Retinoblastoma: MR imaging parameters in detection of tumor extent. Radiology 2005;235: 197-207
3 Schueler AO, Hosten N, Bechrakis NE, et al. High resolution magnetic resonance imaging of retinoblastoma. $\mathrm{Br} \mathrm{J}$ Ophthalmol 2003;87:330-335

4 Honavar SG. Orbital retinoblastoma. In: Singh AD, Damato BE, Pe'er J, Murphree AL, Perry JD, eds. Clinical Ophthalmic Oncology. Philadelphia: W.B. Saunders; 2007:477-483

5 Lyle CE, Rodriguez-Galindo C, Wilson MW. Trilateral retinoblastoma. In: Singh AD, Damato BE, Pe'er J, Murphree AL, Perry JD, eds. Clinical Ophthalmic Oncology. Philadelphia: W.B. Saunders; 2007: 438-440

6 De Potter P. Imaging techniques. In: Singh AD, Damato BE, Pe'er J, Murphree AL, Perry JD, eds. Clinical Ophthalmic Oncology. Philadelphia: W.B. Saunders; 2007:507-516

7 Cunningham FG, Leveno KJ, Bloom SJ, et al. Williams Obstetrics, 22nd ed. New York: McGraw Hill; 2005:349-365

8 Callen PW. Ultrasonography in Obstetrics and Gynecology, 3rd ed. Philadelphia: W.B. Saunders; 1994:8-16

9 Mattison DR, Kay HH, Miller RK, et al. Magnetic resonance imaging: a noninvasive tool for fetal and placental physiology. Biol Reprod 1988;39:39-49

10 Maat-Kievit JA, Oepkes D, Hartwig NG, Vermeij-Keers C, van Kamp IL, van de Kamp JJ. A large retinoblastoma detected in a fetus at 21 weeks of gestation. Prenat Diagn 1993;13:377-384

11 Salim A, Wiknjosastro GH, Danukusumo D, Barnas B, Zalud I. Fetal retinoblastoma. J Ultrasound Med 1998;17:717-720

12 Toi A, Sutherland J, Gallie B, et al. Evaluation of the fetus at risk for retinoblastoma: what is the role of prenatal ultrasound? Ultrasound Med Biol 2003;29:S137

13 Finger PT, Khoobehi A, Ponce-Contreras MR, Rocca DD, Garcia JP Jr. Three dimensional ultrasound of retinoblastoma: initial experience. Br J Ophthalmol 2002;86:1136-1138

14 Singh AD, Balmer A, Munier F. Retinocytoma or retinoma. In: Singh AD, Damato BE, Pe'er J, Murphree AL, Perry JD, eds. Clinical Ophthalmic Oncology. Philadelphia: W.B. Saunders; 2007: 487-490

15 Brisse HJ, Lumbroso L, Fréneaux PC, et al. Sonographic, CT, and MR imaging findings in diffuse infiltrative retinoblastoma: report of two cases with histologic comparison. AJNR Am J Neuroradiol 2001;22:499-504

16 Traboulsi EI, Heur M, Singh A. Tumors of the retinal pigment epithelium. In: Singh AD, Damato BE, Pe'er J, Murphree AL, Perry JD, eds. Clinical Ophthalmic Oncology. Philadelphia: W.B. Saunders; 2007:358-365

17 Singh AD, Traboulsi E, Schoenfield L. Neuro-oculocutaneous syndromes (phakomatoses). In: Singh AD, Damato BE, Pe'er J, Murphree AL, Perry JD, eds. Clinical Ophthalmic Oncology. Philadelphia: W.B. Saunders; 2007:385-401

18 Kliegman RM, Behrman RE, Jenson HB, et al. Nelson Textbook of Pediatrics, 18th ed. W.B. Saunders: Philadelphia; 2007: 2151-2152 